\title{
The path of the superior sagittal sinus in unicoronal synostosis
}

\author{
Aaron J. Russell • Kamlesh B. Patel • Gary Skolnick • \\ Albert S. Woo • Matthew D. Smyth
}

Received: 5 December 2013 / Accepted: 6 February 2014 / Published online: 28 February 2014

(C) Springer-Verlag Berlin Heidelberg 2014

\begin{abstract}
Purpose This study investigates the anatomic relationship between the superior sagittal sinus (SSS) and the sagittal suture in infants with uncorrected unicoronal synostosis. The morphology of the SSS is also evaluated postoperatively to assess whether normalization of intracranial structures occurs following reconstruction.

Methods The study sample consisted of 20 computed tomography scans (10 preoperative, 6 postoperative, and 4 unaffected controls) obtained between 2001 and 2013. The SSS and the sagittal suture were outlined using Analyze imaging software. These data were used to measure the maximum lateral discrepancy between the SSS and the sagittal suture preoperatively and to assess for postoperative changes in the morphology of the SSS.

Results In children with uncorrected unicoronal synostosis, the SSS deviates to the side of the patent coronal suture posteriorly and tends to follow the path of the sagittal and metopic sutures. The lateral discrepancy between the SSS and the sagittal suture ranged from 5.0 to $11.8 \mathrm{~mm}$, with a $99.9 \%$ upper prediction bound of $14.4 \mathrm{~mm}$. Postoperatively, the curvature of the SSS was statistically decreased following surgical intervention, though it remained significantly greater than in unaffected controls.

Conclusions The SSS follows a predictable course relative to surface landmarks in children with unicoronal synostosis.
\end{abstract}

\footnotetext{
A. J. Russell $(\bowtie)$

School of Medicine, Washington University, St. Louis, MO, USA

e-mail: russella@wusm.wustl.edu

K. B. Patel $\cdot$ G. Skolnick $\cdot$ A. S. Woo

Department of Plastic and Reconstructive Surgery, Washington

University School of Medicine, St. Louis, MO, USA

M. D. Smyth

Department of Neurological Surgery, Washington University School

of Medicine, St. Louis, MO, USA
}

When creating burr holes for craniotomies, the SSS can be avoided in $99.9 \%$ of cases by remaining at least $14.4 \mathrm{~mm}$ from the lateral edge of the sagittal suture. Postoperative changes in the path of the SSS provide indirect evidence for normalization of regional brain morphology following frontoorbital advancement.

Keywords Unicoronal synostosis · Superior sagittal sinus · Fronto-orbital advancement

\section{Introduction}

Craniosynostosis is a congenital deformity of the skull caused by the abnormal fusion of one or more calvarial sutures. It has an estimated prevalence of $1: 2,000$ to 1:2,500 live births $[3,9$, 13] and produces a characteristic set of cranial abnormalities based on the specific suture involved. Unicoronal synostosis (UCS), defined by the premature fusion of a single coronal suture, is the third most common form of craniosynostosis [8, 14]. It occurs more frequently in females and predominantly affects the right coronal suture $[8,9,14]$. Individuals with UCS present with anterior plagiocephaly, an abnormal cranial morphology characterized by flattening of the ipsilateral forehead, protrusion of the contralateral forehead, and elevation of the eyebrow and superior orbital rim on the ipsilateral side $[2$, $11]$.

Fronto-orbital advancement is the primary surgical intervention for the treatment of anterior plagiocephaly. It is an open procedure that involves unilateral or bilateral advancement of the fronto-orbital bandeau, a horizontal strip of bone encompassing the superior rim of both orbits $[5,11,12]$. In order to obtain surgical access for this procedure, a frontal craniotomy is performed. Following the initial dissection of soft tissue from the calvaria, burr holes are drilled on either side of the anterior fontanelle to allow epidural access for 
Table 1 Study sample: age at preoperative and postoperative scan

\begin{tabular}{|c|c|c|c|c|c|c|c|}
\hline \multicolumn{3}{|c|}{ Right unicoronal synostosis $(N=5)$} & \multicolumn{3}{|c|}{ Left unicoronal synostosis $(N=5)$} & \multicolumn{2}{|c|}{ Normal controls $(N=4)$} \\
\hline $\begin{array}{l}\text { Preop age } \\
\text { (months) }\end{array}$ & $\begin{array}{l}\text { Postop age } \\
\text { (months) }\end{array}$ & Sex & $\begin{array}{l}\text { Preop age } \\
\text { (months) }\end{array}$ & $\begin{array}{l}\text { Postop age } \\
\text { (months) }\end{array}$ & Sex & $\begin{array}{l}\text { Age } \\
\text { (months) }\end{array}$ & Sex \\
\hline 2 & - & $\mathrm{M}$ & 0.5 & 16.5 & $\mathrm{~F}$ & 3 & M \\
\hline 2 & - & $\mathrm{F}$ & 3 & - & $\mathrm{F}$ & 4 & M \\
\hline 3 & 18 & $\mathrm{M}$ & 4.5 & - & $\mathrm{F}$ & 6 & $\mathrm{~F}$ \\
\hline 4 & 17.5 & $\mathrm{M}$ & 6.5 & 20.5 & $\mathrm{M}$ & 18 & $\mathrm{~F}$ \\
\hline 5 & 13.5 & $\mathrm{~F}$ & 8.5 & 35 & M & & \\
\hline
\end{tabular}

craniotomy. While complications with this step are relatively uncommon, the superior sagittal sinus (SSS) is at risk of injury due to its proximity to the fontanelle. In healthy children, mean blood flow through the SSS is approximately $0.5 \mathrm{~L} /$ min [7]. In turn, injury to the SSS could lead to massive hemorrhaging and even death. Due to such risks, great care is taken to avoid insult to this structure. Neurosurgeons will commonly place their burr holes just lateral to the midline of the skull, as defined by the sagittal suture. However, when significant frontal asymmetry exists (as in the case of UCS), the location of the SSS becomes unclear, and its relationship to the sagittal suture cannot be presumed.

This study is designed to better characterize the location of the SSS relative to cranial surface landmarks in children with UCS. Computed tomography (CT) scans are analyzed to define the size, shape, and path of both the SSS and the sagittal suture. The morphology of the endocranial base is also examined to investigate its influence on the SSS. The goal of this study is to enhance our understanding of dural venous anatomy in children with craniosynostosis in order to better equip surgeons who operate on these patients. A secondary objective

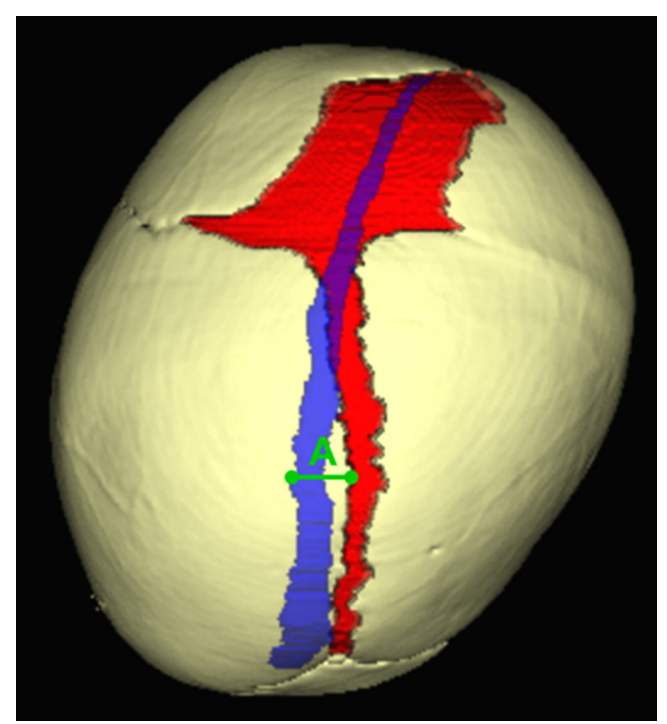

Fig. 1 Maximum discrepancy between the sagittal suture and the superior sagittal sinus. A: Sample measurement (superior sagittal sinus-blue, sagittal suture-red) is to compare pre- and postoperative CT scans of children with UCS to assess for changes in brain morphology following surgical reconstruction with fronto-orbital advancement.

\section{Methods}

Imaging data consisted of preoperative CT scans from 10 infants with isolated UCS, ranging in age from 0.5 to 8.5 months (mean, 3.9 months, Table 1). From 163 patients seen between 1981 and 2013, we selected 10 cases with excellent preoperative data quality that were equally distributed between right and left UCS. Scan sets with incomplete data, motion artifacts, or other imaging defects were excluded. Postoperative scans were obtained from six of these children at approximately 1 year post-repair. These patients had undergone fronto-orbital advancement at an average age of 6.8 months (range, 4.5 to 9 months) and were re-scanned at

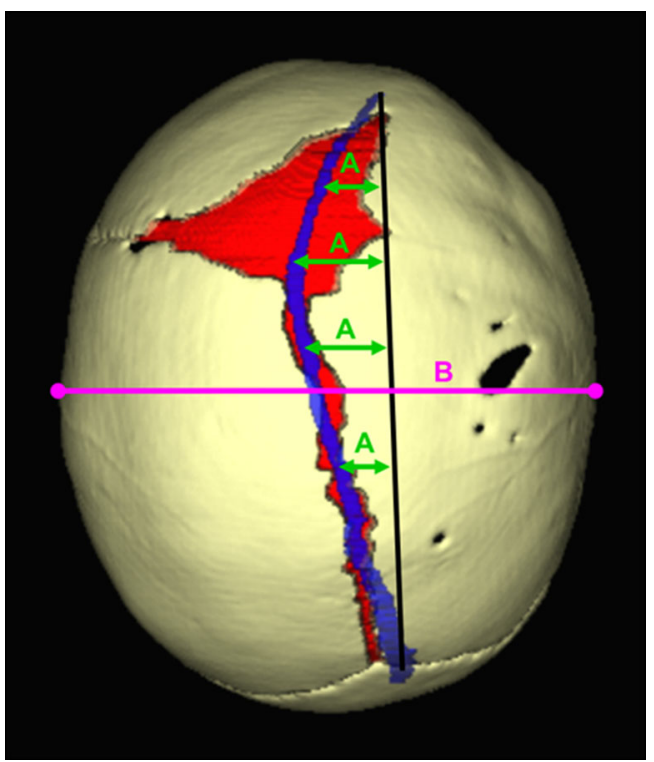

Fig. 2 Quantitative measurement of curvature. $A$ Sample lines showing the distance from the center of the superior sagittal sinus to a straight line connecting the anterior and posterior ends of the sinus. $B$ Maximum cranial width. Deviation score $=$ mean $(A) / B \times 100$ (superior sagittal sinus—blue, sagittal suture—red) 
Fig. 3 a Cribriform plate angle. $\theta 1$ : Angle between the midlines of the cribriform plate and the foramen magnum. b Petrous ridge asymmetry. $\theta 2$ : Angle between the petrous ridge and the midline of the cribriform plate on the synostotic side. $\theta 3$ : Angle between the petrous ridge and the cribriform plate on the unaffected side. Petrous ridge asymmetry $=\theta 3-\theta 2$
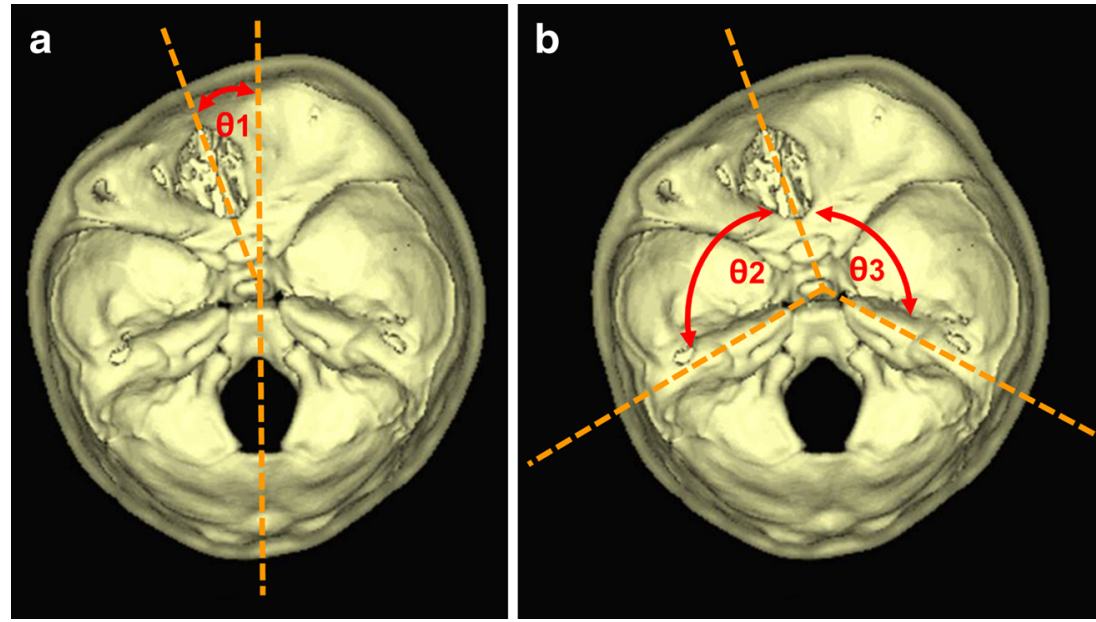

an average age of 20.2 months (range, 13.5 to 35 months). The duration of time between preoperative and postoperative scans ranged from 8.5 to 26.5 months (average, 15.6 months). CT scans were acquired at St. Louis Children's Hospital between November 2001 and June 2013 as part of the routine evaluation and follow-up of infants with UCS. All imaging analyses were performed following Institutional Review Board approval. Subjects were excluded if they demonstrated multiple suture synostosis or other craniofacial anomalies. This study also included four age-matched controls. These children had undergone craniofacial CT scans for various reasons but were unaffected by craniosynostosis. Those with a history of craniomaxillofacial fracture, surgical intervention, or developmental abnormalities were excluded.

Analyze 11.0 software (Mayo Clinic, Rochester, MN) was utilized to define the size, shape, and path of the superior
Fig. 4 Reliability test. Vertex view of two subjects with right unicoronal synostosis. $a$ Initial analysis. $b$ Analysis performed 1 week later (superior sagittal sinus - blue, sagittal suture — red)
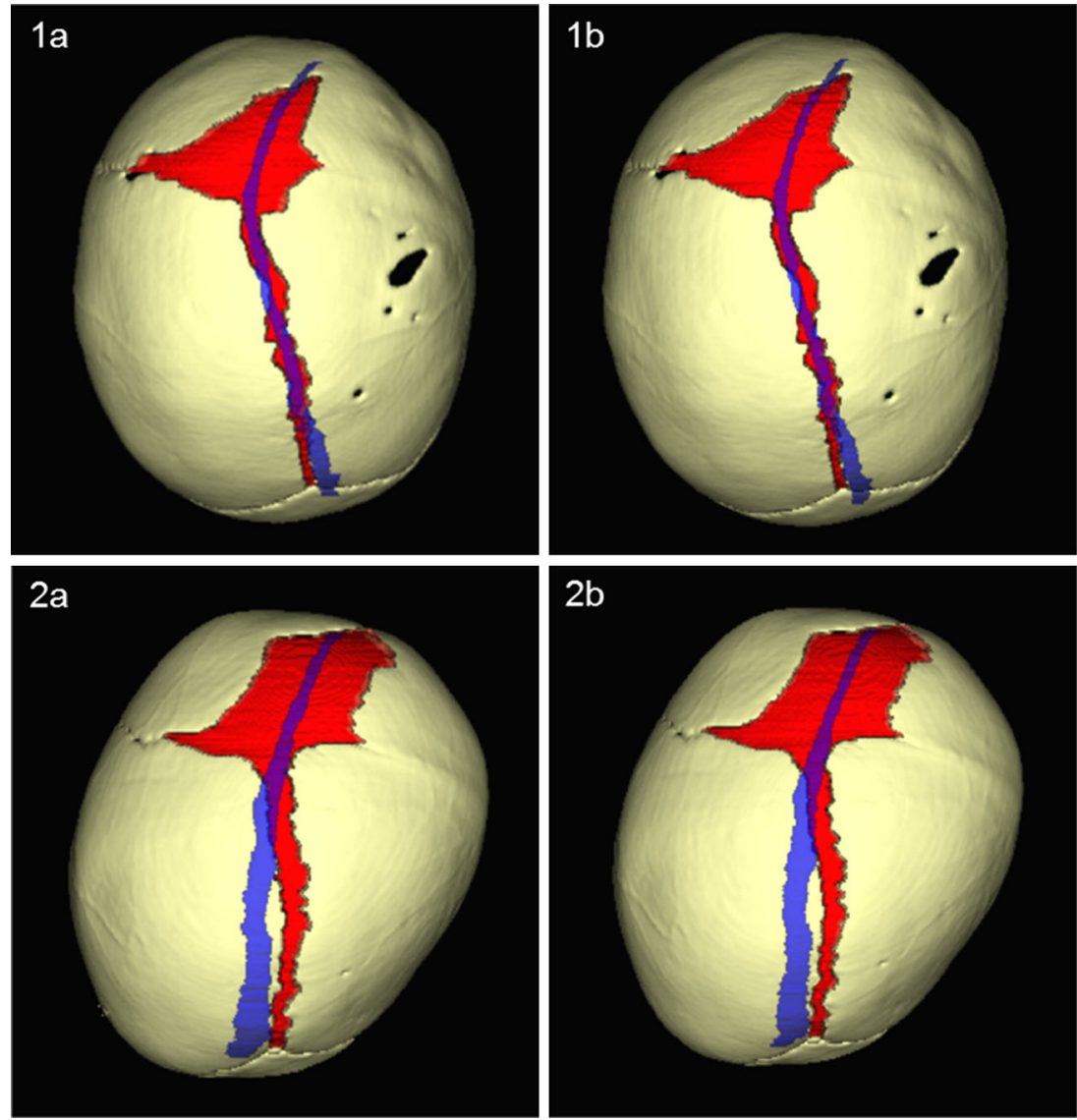
sagittal sinus and the sagittal suture. CT data were translated into three-dimensional reconstructions and oriented to the Frankfort horizontal plane using a bony threshold. Anteriorposterior alignment was defined using the basion and opisthion. The re-oriented skull was then broken down into $0.5 \mathrm{~mm}$ slices. The Image Edit module was used to manually trace the SSS and sagittal suture on consecutive coronal slices. After delineating the structures slice-by-slice, the skull and segmented components were converted into individual threedimensional objects using the Volume Edit module. Only the SSS was labeled in postoperative scans due to the absence of the sagittal suture post-repair. To evaluate for reliability of the data, the protocol was repeated on two randomly selected preoperative CT scans. The second analysis was performed by the same entity 1 week after the initial evaluation. Due to the limitations in assessing the shape of a structure threedimensionally, no quantitative comparison was performed. Instead, consistency was assessed through visual inspection of both three-dimensional images.

The position and curvature of the SSS were characterized using two quantitative measures. First, we determined the maximum lateral discrepancy between the SSS and the sagittal suture in infants with uncorrected UCS. Distances were measured from the edge of the sagittal suture to the far edge of the SSS (Fig. 1). The point of maximum discrepancy was found by performing multiple measurements along the length of the sinus. Measurements were made on two separate occasions. Average values were used in calculating a $99.9 \%$ prediction interval. To assess curvature, we calculated the mean lateral distance between a curve running through the center of the SSS and a straight line connecting the anterior and posterior ends of the sinus (Fig. 2). To do so, the Region of Interest module was used to determine the geometric center of the SSS in consecutive coronal slices. The mean distance between the sinus and the line was calculated. Final values were normalized to maximum cranial width to account for variations in head size.

Abnormalities of the cranial base were examined in all subjects using the same pre- and postoperative CT scan reconstructions. First, the midlines of the cribriform plate and the foramen magnum were defined using the Volume Render module of Analyze. The intersection of these lines was used to measure the angle of deflection between the anterior and posterior fossae (Fig. 3a). Next, we examined the alignment of the cribriform plate relative to the petrous ridge of the temporal bone. Angles were measured from the petrous ridge to the cribriform plate bilaterally, and the difference between the normal and synostotic sides was used as a marker of cranial base asymmetry (Fig. 3b). Student's $t$ tests were employed to compare groups. $p$ values less than 0.05 were
Fig. 5 1-4 Vertex view of four control subjects unaffected by craniosynostosis (superior sagittal sinus - blue, sagittal suture - red)
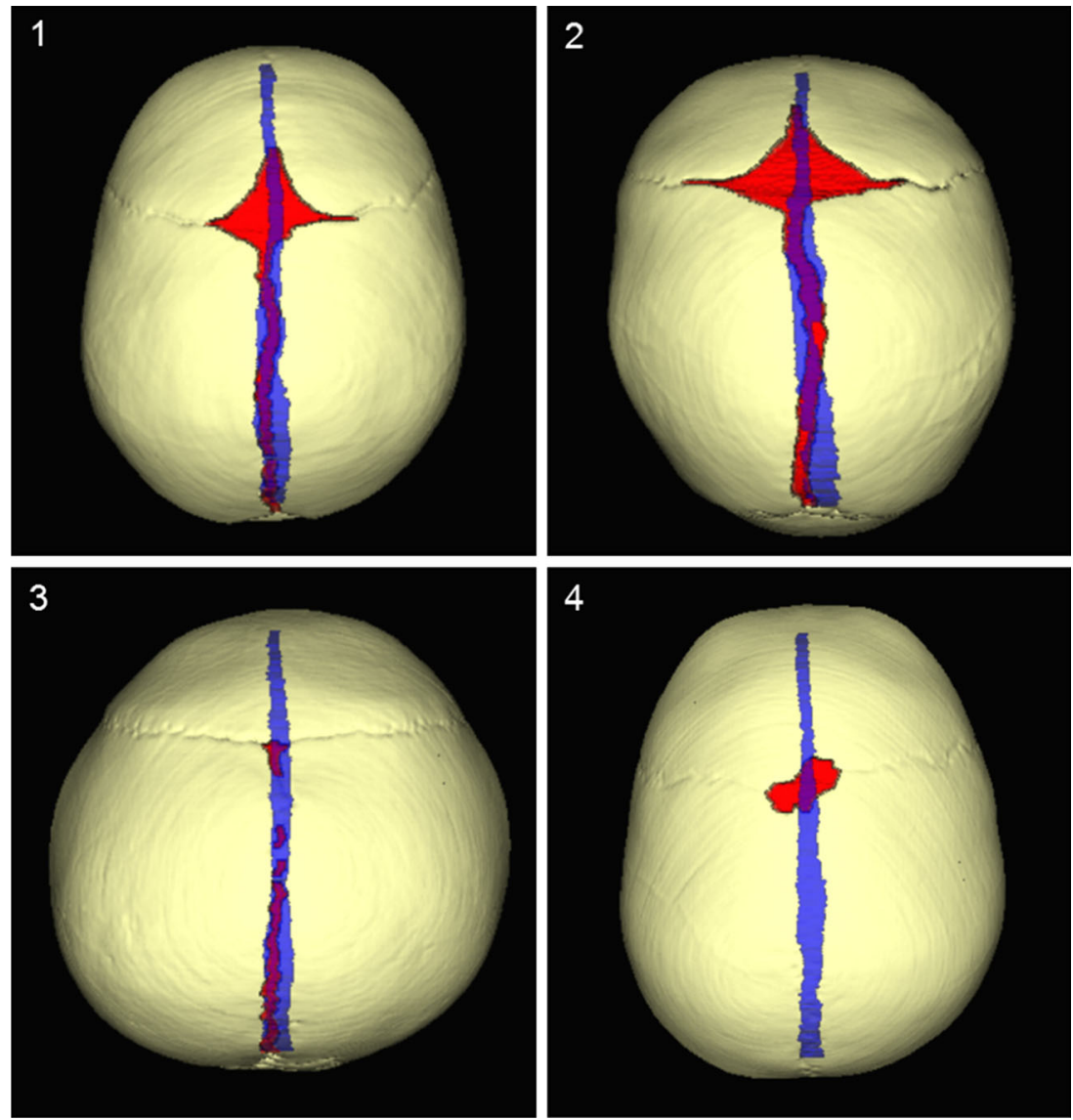
considered significant. Correlations between variables were assessed using Pearson's $r^{2}$. All statistical calculations were performed using Excel 2010 (Microsoft, Redmond, WA).

\section{Results}

Reliability

Figure 4 shows the results of the repeat analysis of two preoperative CT scans. The paths of the superior sagittal sinus and the sagittal suture are nearly identical, demonstrating excellent intra-rater reliability. Figure 5 confirms that the path of the SSS is essentially linear in unaffected children and lies directly inferior to the sagittal suture prior to sutural closure.

Preoperative findings

Preoperative CT scan reconstructions of 10 infants with uncorrected UCS are presented in Fig. 6 (right UCS) and Fig. 7 (left UCS). In these children, the SSS travels an arc roughly in line with the sagittal and metopic sutures. Anteriorly, the sagittal sinus runs closely with the metopic suture as it travels toward the nasion. Posteriorly, its relationship to the sagittal suture is more variable as the sinus tends to deviate toward the side of the patent coronal suture. This pattern is observed in 9/10 subjects, the exception being one infant with right UCS whose sagittal sinus lies slightly to the ipsilateral side of the premature fusion (image 5 in Fig. 6).

In all 10 subjects, the largest discrepancy between the SSS and the sagittal suture was located posterior to bregma. In 8/10 children, the point of maximum deviation was contralateral to the synostosis. This distance, measured from the edge of the sagittal suture to the far edge of the sinus, ranged from 5 to $11.8 \mathrm{~mm}$ with a mean value of $8.0 \mathrm{~mm}$ and a standard deviation of $2.0 \mathrm{~mm}$. Statistical analysis revealed a $99.9 \%$ upper prediction bound of $14.4 \mathrm{~mm}$. There was no significant correlation between age and the size of the maximum discrepancy (Pearson's $r^{2}=0.075, p=0.45$ ).

\section{Postoperative changes}

Figure 8 shows the path of the SSS before and after surgical correction of UCS in six subjects. The curvature of the SSS,
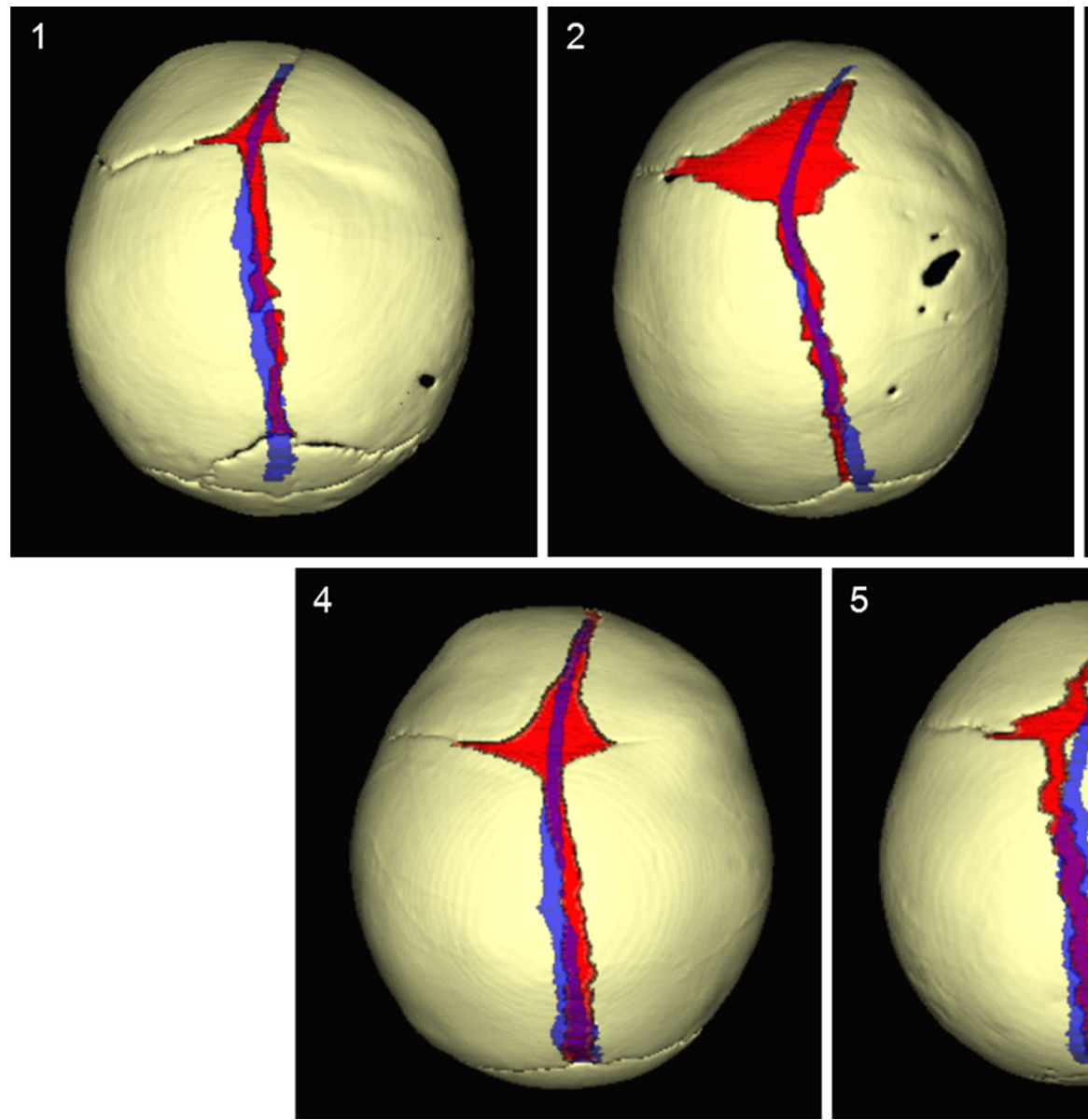

Fig. 6 1-5 Vertex view of five subjects with right unicoronal synostosis (superior sagittal sinus - blue, sagittal suture-red)
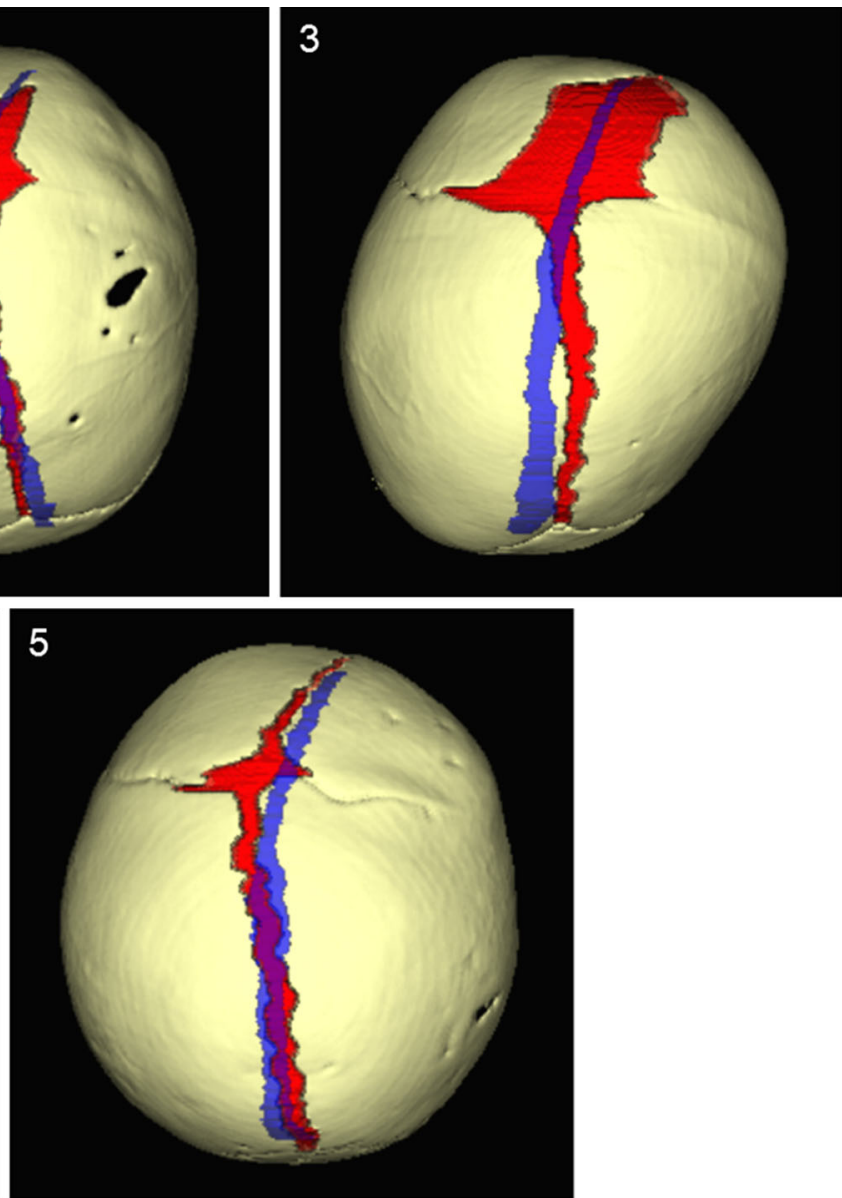

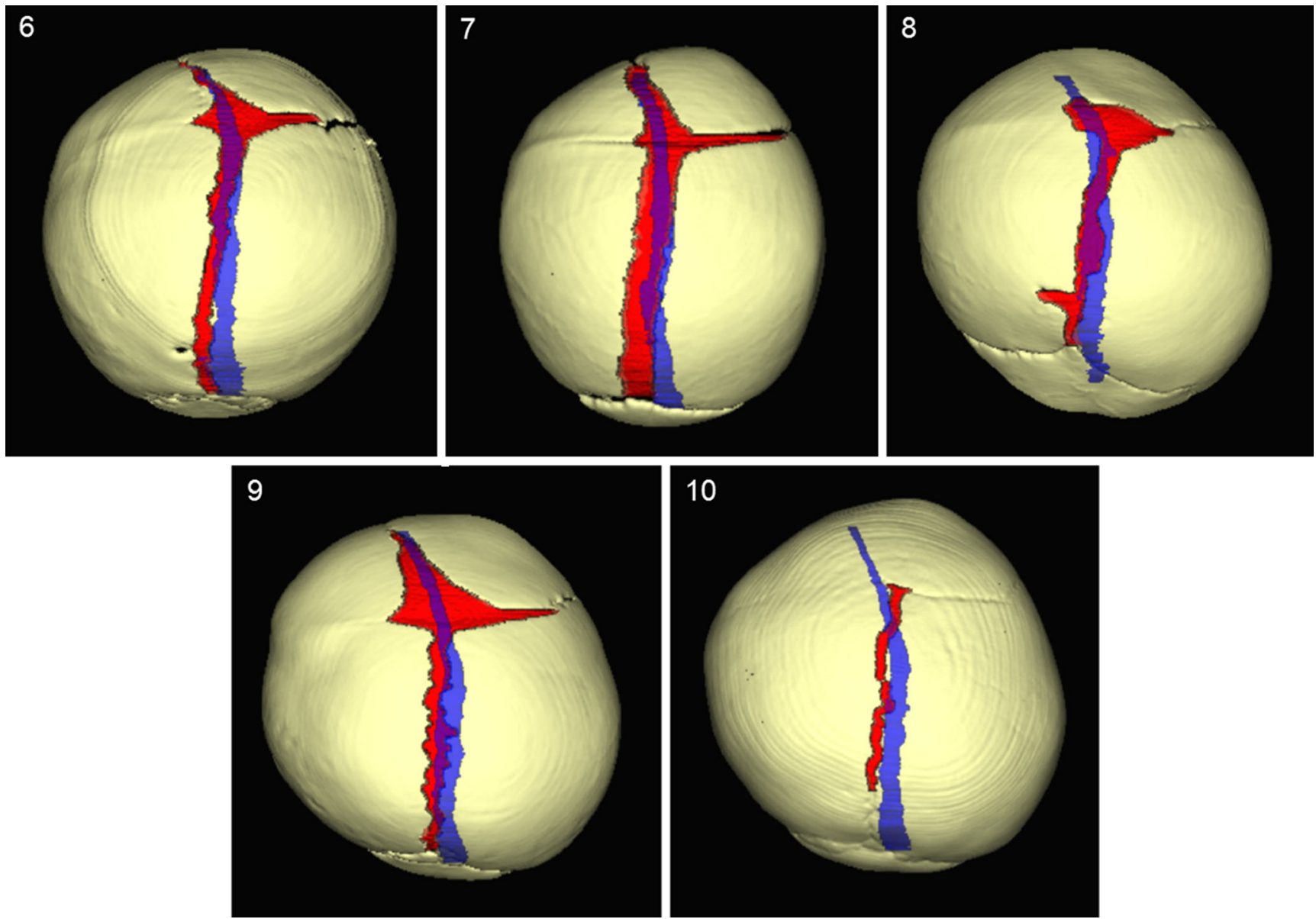

Fig. 7 6-10 Vertex view of five subjects with left unicoronal synostosis (superior sagittal sinus— blue, sagittal suture-red)

calculated as the mean lateral distance from a straight line, had the following ranges: 2.4 to $12.1 \mathrm{~mm}$ in preoperative cases (mean $=6.0 \pm 0.8 \mathrm{~mm}$ ), 0.6 to $7.1 \mathrm{~mm}$ in postoperative cases $(4.2 \pm 0.9 \mathrm{~mm})$, and 0.5 to $1.5 \mathrm{~mm}$ in controls $(0.9 \pm 0.2 \mathrm{~mm})$. As a percentage of maximum cranial width, these ranged from 2.5 to $10.4 \%$ preoperatively (mean $=5.2 \pm 0.7 \%$ ), 0.5 to $5.3 \%$ postoperatively ( $3.0 \pm 0.7 \%$ ), and 0.6 to $1.3 \%$ in controls ( 0.8 $\pm 0.2 \%$ ) (Fig. 9). Postoperative curvature of the SSS was significantly reduced from pre-repair curvature when normalized to maximum cranial width $(p=0.037)$. The curvature of the SSS was also significantly less in controls than for both the preoperative $(p=0.003)$ and postoperative $(p=0.025)$ cases.

\section{Cranial base measures}

The angle between the cribriform plate and the foramen magnum in patients with uncorrected UCS ranged from $10.8^{\circ}$ to $33.4^{\circ}\left(\right.$ mean $\left.=18.8^{\circ} \pm 2.0^{\circ}\right)$. This angle ranged from $3.7^{\circ}$ to $23.3^{\circ}\left(14.3^{\circ} \pm 3.0^{\circ}\right)$ in subjects following cranial vault reconstruction and from $0.8^{\circ}$ to $3.8^{\circ}\left(2.2^{\circ} \pm 0.6^{\circ}\right)$ in subjects unaffected by craniosynostosis (Fig. 10a). The cribriform plate angle was significantly smaller in normal controls than in both preoperative $(p<0.001)$ and postoperative $(p=0.009)$ subjects. The reduction in the cribriform plate angle following fronto-orbital advancement was not statistically significant $(p=0.123)$. Petrous ridge asymmetry, measured as the difference between the two petrous ridge angles, ranged from $24.5^{\circ}$ to $78.6^{\circ}\left(45.8^{\circ} \pm 5.1^{\circ}\right)$ in preoperative scans. This difference ranged from $14.1^{\circ}$ to $71.5^{\circ}\left(40.2^{\circ} \pm 8.4^{\circ}\right)$ in postoperative scans and from $1.0^{\circ}$ to $15.1^{\circ}\left(6.1^{\circ} \pm 3.3^{\circ}\right)$ in normal controls (Fig. 10b). Petrous ridge asymmetry was significantly reduced following surgical reconstruction $(p=0.009)$. The cranial base in unaffected children was significantly more symmetric than both the preoperative $(p<0.001)$ and postoperative $(p=0.008)$ groups.

Using the normalized bend measures of the SSS, we found that the curvature of the SSS was not significantly correlated with either the cribriform plate angle $\left(r^{2}=0.007, p=0.820\right)$ or the petrous ridge asymmetry $\left(r^{2}=0.084, p=0.418\right)$ in infants with uncorrected UCS. Postoperatively, there was a positive but not significant correlation between the curvature of the SSS and the petrous ridge asymmetry $\left(r^{2}=0.460, p=0.139\right)$. There was a significant strong positive correlation between the 

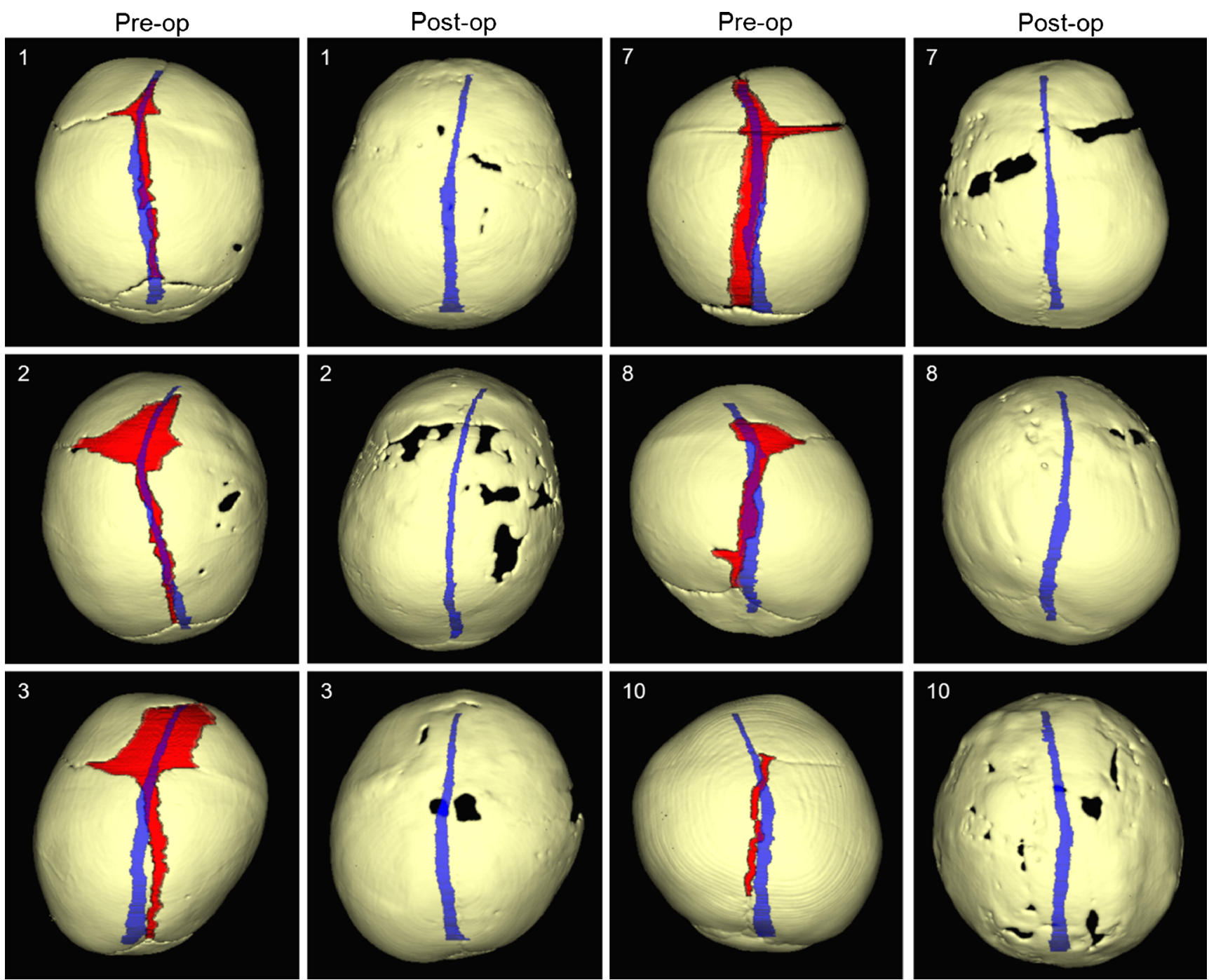

Fig. $81-3,7,8,10$ Vertex view of six subjects before and after cranial vault remodeling (superior sagittal sinus—blue, sagittal suture—red)

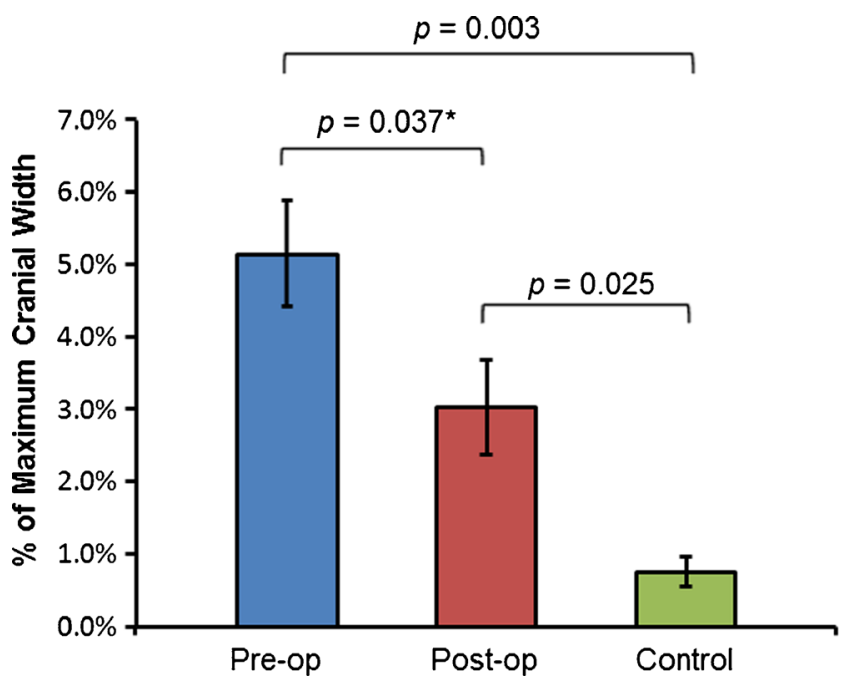

Fig. 9 Mean curvature of the SSS normalized to maximum cranial width. Curvature was defined as the mean lateral distance between a curve running through the center of the SSS and a straight line connecting the anterior and posterior ends of the sinus. ${ }^{*} p$ value derived from paired $t$ test comparing only those subjects with both pre- and postoperative data

cribriform plate angle and the deformity of the SSS post-repair $\left(r^{2}=0.870, p=0.007\right)$

\section{Discussion}

The sagittal suture is an important surface landmark in children undergoing cranial vault reconstruction. The suture defines the junction between the two parietal bones and typically occupies the midline of the skull from lambda to the anterior fontanelle. In normal infants, this structure is roughly collinear with the metopic suture. However, in children with anterior plagiocephaly, this relationship is distorted due to the significant frontal asymmetry. Rather than forming a straight line, the sagittal and metopic sutures meet at an obtuse angle.

Alterations in the surface anatomy of the skull are reflected in the intracranial vasculature. As seen in the threedimensional reconstructions of children with uncorrected UCS, the superior sagittal sinus travels from lambda to the 
Fig. 10 Cribriform plate angle (a) and petrous ridge asymmetry (b) measured in preoperative, postoperative, and control subjects. ${ }^{*} p$ value derived from paired $t$ test comparing only those subjects with both pre- and postoperative data
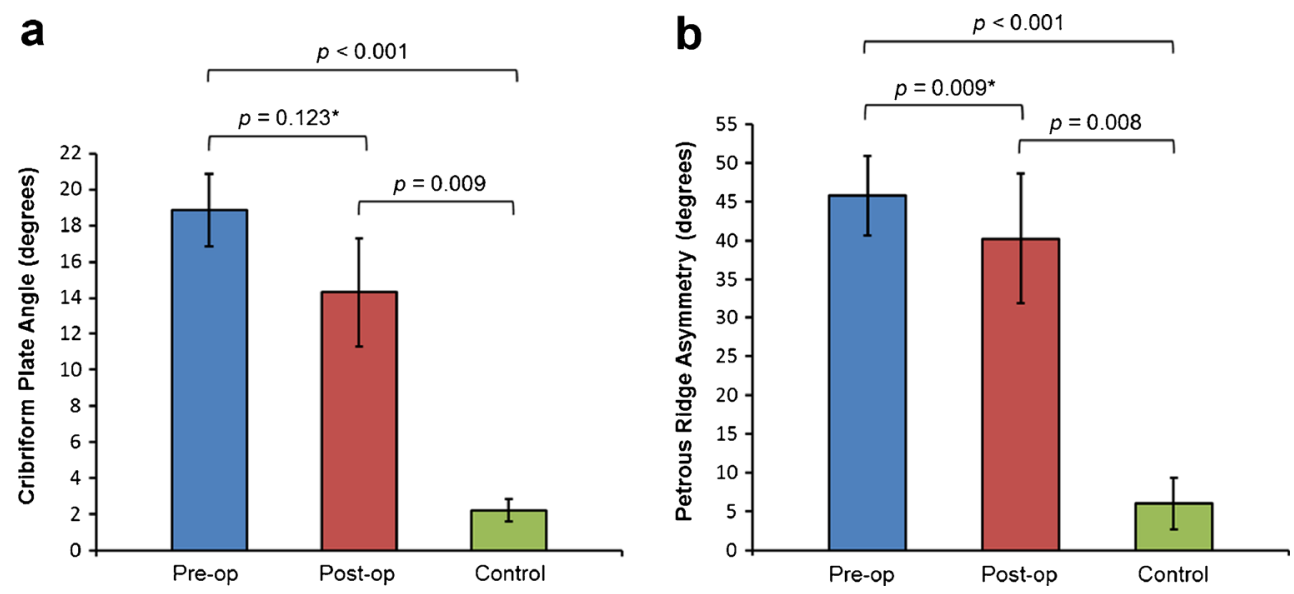

anterior fontanelle and then bends with the metopic suture as it continues anteriorly. The deformity of the SSS is suggestive of alterations in regional brain morphology. Aldridge et al. describe several morphological variations in children with UCS, including anteroposterior shortening of the frontal lobe ipsilateral to the fused coronal suture [1]. These changes are not associated with reductions in intracranial volume or whole brain volume [6]. In $99.9 \%$ of UCS patients, the maximum lateral discrepancy between the SSS and the sagittal suture is predicted to be less than or equal to $14.4 \mathrm{~mm}$. This number is particularly useful for positioning burr holes for craniotomy. By remaining $14.4 \mathrm{~mm}$ or farther from the edge of the sagittal suture, the surgeon can safely avoid the SSS in the vast majority of patients. It should be noted that the largest discrepancies were measured posterior to bregma in all 10 patients. Although the maximum deviation was typically found on the nonsynostotic side, in 2/10 cases, it was located ipsilateral to the premature fusion. Therefore, this prediction is applicable to both sides of the sagittal suture.

Fronto-orbital advancement has clear benefits in relieving the craniofacial deformities seen in patients with anterior plagiocephaly. However, the quality of the surgical outcomes is highly dependent on the initial severity of the skull base abnormalities [4]. Di Rocco and Velardi developed a threestage classification system for patients with unilateral anterior plagiocephaly. Type III anterior plagiocephaly, the most severe form, is characterized by contralateral deviation of the nasal pyramid, ipsilateral deviation of the vomer bone, and anterior displacement of the petrous ridge on the ipsilateral side. Patients with these findings typically have the poorest surgical outcomes [4].

Our analysis of cranial base changes in patients with anterior plagiocephaly revealed significant improvements in the symmetry of the anterior and middle cranial fossae following fronto-orbital advancement. The angle between the anterior and posterior fossae was also reduced by an average of $4.5^{\circ}$, though this change was statistically insignificant. Our results corroborate the findings of Marsh and Vannier, who reported increased symmetry of the anterior fossa along with minor changes in the anterior-posterior axis following fronto-orbital advancement [10]. Overall, there is a small but significant improvement in cranial base morphology following surgical intervention, although the cranial base remains significantly less symmetrical than in normal controls.

Alterations in the path of the SSS were observed following fronto-orbital advancement. Postoperative curvature of the SSS was statistically smaller than preoperative values but was still significantly greater than normal controls. This suggests that hemispheric symmetry improves following surgical intervention but remains substantially different relative to unaffected children. In patients with uncorrected UCS, there was no correlation between the curvature of the SSS and the severity of the skull base deformities. Consequently, the classification of anterior plagiocephaly advanced by Di Rocco and Veraldi [4] does not reflect changes in the SSS, as the curvature of the SSS appears to be independent of cranial base abnormalities. A more reliable predictor of sagittal sinus morphology is the overlying calvarial sutures. Postoperatively, there was a significant correlation between the cribriform plate angle and the curvature of the SSS. We do not have a good morphological explanation for this result; one possibility is that this positive correlation is a type I error, the result of random variation among the limited number of postoperative cases.

As in all retrospective analyses, this study suffers from a number of weaknesses, the greatest of which is the small sample size. In total, we examined 10 children with uncorrected UCS, 6 after cranial reconstruction, and 4 age-matched controls. Despite this limitation, however, we were able to statistically establish a $99.9 \%$ confidence interval for the distance between the sagittal suture and the SSS. Regardless, additional data may serve to further increase the accuracy of our predictive model. In addition, this study did not (and could not) evaluate brain morphology directly. Instead, we measured changes in the curvature of the SSS. Further investigations to assess postoperative morphology using more direct methods 
(e.g., MRI) might better characterize changes in the frontal and parietal lobes.

In summary, the SSS in children with uncorrected UCS follows a path approximately in line with the sagittal and metopic sutures and is independent of deformities of the endocranial base. Typically, the sinus will deviate slightly away from the synostosis and toward the patent coronal suture. This has practical implications for the surgical repair of UCS. When performing craniotomies, the risk of compromising the SSS is significantly reduced when burr holes are drilled $14.4 \mathrm{~mm}$ or farther from the outer edge of the sagittal suture, especially on the nonsynostotic side. Comparisons of the SSS pre- and postoperatively also provide indirect evidence that fronto-orbital advancement may have a beneficial effect on regional brain morphology and hemispheric symmetry.

Acknowledgments Research reported in this publication was supported by the St. Louis Children's Hospital Foundation - Children's Surgical Sciences Institute.

\section{References}

1. Aldridge K, Kane AA, Marsh JL, Panchal J, Boyadjiev SA, Yan P, Govier D, Ahmad W, Richtsmeier JT (2005) Brain morphology in nonsyndromic unicoronal craniosynostosis. Anat Rec A Discov Mol Cell Evol Biol 285(2):690-698. doi:10.1002/ar.a.20201

2. Bernhardt P, Lindström A, Maltese G, Tarnow P, Lagerlöf JH, Kölby L (2013) A novel quantitative image-based method for evaluating cranial symmetry and its usefulness in patients undergoing surgery for unicoronal synostosis. J Craniofac Surg 24(1):166-169. doi:10. 1097/SCS.0b013e31826468bc
3. Boulet SL, Rasmussen SA, Honein MA (2008) A population-based study of craniosynostosis in metropolitan Atlanta, 1989-2003. Am J Med Genet 146A(8):984-991. doi:10.1002/ajmg.a.32208

4. Di Rocco C, Velardi F (1988) Nosographic identification and classification of plagiocephaly. Childs Nerv Syst 4(1):9-15

5. Fearon JA (2008) Beyond the bandeau: 4 variations on fronto-orbital advancements. J Craniofac Surg 19(4):1180-1182. doi:10.1097/SCS. 0b013e3181764b3e

6. Hill CA, Vaddi S, Moffitt A, Kane AA, Marsh JL, Panchal J, Richtsmeier JT, Aldridge K (2011) Intracranial volume and whole brain volume in infants with unicoronal craniosynostosis. Cleft Palate Craniofac J 48(4):394-398. doi:10.1597/10-051

7. Hirabuki N, Watanabe Y, Mano T, Fujita N, Tanaka H, Ueguchi T, Nakamura H (2000) Quantitation of flow in the superior sagittal sinus performed with cine phase-contrast MR imaging of healthy and achondroplastic children. AJNR Am J Neuroradiol 21(8):1497-1501

8. Kolar JC (2011) An epidemiological study of nonsyndromal craniosynostoses. J Craniofac Surg 22(1):47-49. doi:10.1097/SCS. 0b013e3181f6c2fb

9. Lajeunie E, Le Merrer M, Bonaïti-Pellie C, Marchac D, Renier D (1995) Genetic study of nonsyndromic coronal craniosynostosis. Am J Med Genet 55(4):500-504. doi:10.1002/ajmg.1320550422

10. Marsh JL, Vannier MW (1986) Cranial base changes following surgical treatment of craniosynostosis. Cleft Palate J 23(Suppl 1):918

11. Matushita H, Alonso N, Cardeal DD, de Andrade F (2012) Frontalorbital advancement for the management of anterior plagiocephaly. Childs Nerv Syst 28(9):1423-1427. doi:10.1007/s00381-012-1765-1

12. Pellerin P, Calibre C, Vinchon M, Dhellemmes P, Wolber A, Guerreschi P (2012) Unicoronal synostotic plagiocephaly: surgical correction: Lille's technique. Childs Nerv Syst 28(9):1433-1438. doi:10.1007/s00381-012-1793-x

13. Singer S, Bower C, Southall P, Goldblatt J (1999) Craniosynostosis in Western Australia, 1980-1994: a population-based study. Am J Med Genet 83(5):382-387. doi:10.1002/(SICI)1096-8628(19990423) 83:5<382::AID-AJMG8>3.0.CO;2-A

14. van Veelen-Vincent ML, Mathijssen I, Arnaud E, Renier D, Di Rocco F (2010) Craniosynostosis. In: Lumenta CB, Di Rocco C, Haase J, Mooij JJ (eds) Neurosurgery. Springer, Berlin, pp 501-528 